\title{
Nachlassverzeichnis eines Apothekers in Pernau am Anfang des 18. Jahrhunderts
}

\author{
Raimo Pullat
}

Vor einigen Jahren hat der hier Unterschriebene die Forschungsrichtung im Gebiet dem materiellen Kultur der estnischen Städte in der Frühneuzeit gegründet. Dies bezieht sich vor allem auf das fenomenologische Interpretieren und auf die quantitative und inhaltliche Analyse.

Über die grössere Estnische Städte - Tallinn (Revel), Tartu (Dorpat), Pärnu (Pernau) und Narva ist eine große Menge von Nachlassverzeichnissen erhalten geblieben. Die Tatsache ermöglicht uns, in Bezug auf die Städte und auf die professionelle Gruppen, die miteinander zu vergleichen. Bis hier sind 94 Nachlassverzeichnisse der deutschen Kaufleute in Tallinn a us dem 18. Jahrhundert veröffentlicht w orden [1]. D azu kommen noch eine E dition d er M agisterarbeit und eine Reihe von Beiträge [2]. Zunächst wird die Arbeit von Frau Helve Russak "Die Garderobe der Kaufleute in Tallinn in der e rsten Hälfte des 18. Jah rhunderts" veröffentlicht. Im Jahre 2005 ist eine größere Quellenpublikation der Nachlassverzeichnisse der Stadtbewohner Pernau im 18. Jahrhundert erschienen [3]. Dies demonstriert uns auch die Kontakte zwischen Riga und Pernau. In dieser Forschungsrichtung hat sich eine enge Zusammenarbeit mit Prof. Gerhard Jaritz (Krems), mit Prof. Andrzej Klonder (Warschau), mit Prof. Václav Bůžek (České Budejovice) und mit anderen Wissenschaftlern entwickelt. Der Verfasser ist auch Hauptteilnehmer in dem internationalen großen Projekt "Städtesystem und Urbanisierung im Ostseeraum in der Frühneuzeit" (Rostock). Im Rahmen von diesem Projekt wird ein Teil von publizierten Nachlassverzeichnissen von Tallinn/Revel und Pernau auch digitalisiert.

Nachlassverzeichnisse haben in der Medizingeschichte leider keine ausreichende Anwendung und keine Analyse gehabt. Der Autor ist der Meinung, dass es in diesem Bereich noch viele Entdeckungsmöglichkeiten gibt. In Kollektionen der Nachlassverzeichnisse trifft man di e Na chlassverzeichnisse vo n St adtärzten, Chirurgen, Apothekern, Hebammen und von anderen Medizinern. Diese Nach- 
lassverzeichnisse geben uns neue Informationen über die materielle und geistige Kultur und über die Mentalität der Mediziner. Aus diesem Grunde ist die Veröffentlichung der Quelle im Druck auch begründet. Auch schafft so eine innovative Annäherung der Quellen gute und neue Möglichkeiten für die Zusammenarbeit zwischen Historiker und Mediziner.

Die Nachlassverzeichnisse von Apotheker Gabriel Lütkejohann interessieren die Medizin- und Kulturhistoriker aus mehren Gesichtspunkten. Aus den Nachlassverzeichnissen stellt sich die Lebensweise des Apothekers, die materielle und geistige Kultur von Geld bis Bücher, und von Medikamenten bis Apothekenwaren und Instrumenten heraus. Aus dem Bücherverzeichnis stellt sich der Anteil der theologischen und der weltlichen Literatur heraus. G. Lütkejohann stammte aus Deutschland und hatte Medizin studiert. Aus dem Ratsprotokoll erweist sich, dass er am 1. November im Jahre 1702 den Rat um eine Genehmigung für Apothekengründung in Pernau gebeten hat. 1706-1710 hat er eine Feldapotheke in Rīga geführt. Im November 1710, ein Jahr nach der Eroberung der Stadt Pernau von Russischen Heer, hat er seine Apotheke nach Pernau gezogen. In einem Jahr wurde er Ratsherr, und ziemlich bald - am 29. Mai 1714, ist er gestorben [4].

\section{Anmerkungen}

1. Die Nachlassverzeichnisse der deutschen Kaufleute in Tallinn. Bd. I: 1702-1750 (Tallinn, 1997); Bd. II: 1752-1775 (Tallinn, 2002); Bd. III: 1777-1800 (Tallinn, 2004), bearb. von Raimo Pullat.

2. Lauri Suurmaa, Tallinna saksa kaupmeeste varaloendites inventeeritud laua- ja kööginõud kultuuriajaloo allikana 18. sajandil. In: Vana Tallinn XV (XIX), Tallinn 2004, S. 9-154; Raimo Pullat, Raamat ja lugeja Tallinnas 18. sajandil. In: Vana Tallinn II (VI), Tallinn 1992, S. 80-99; Raimo Pullat, Ex archivo civitatis Revaliensis. In: Vana Tallinn II (VI), Tallinn 1992, S. 152-158; Raimo Pullat, Linnamuusik Ernst Jacob Tewese varaloend. In: Vana Tallinn III (VII), Tallinn 1993, S. 107-117; Raimo Pullat, Hobuseraua- ja sõjariistameistri Johann Georg Engelhardti varaloend. In: Vana Tallinn VII (XI), Tallinn 1997, S. 144-156; Raimo Pullat, 18. sajandi tallinlaste varaloendites sisalduvaid raamatuid. In: Vana Tallinn VIII (XII), Tallinn 1998, S. 173-195; Raimo Pullat, Buch und Leser im Reval des 18. Jahrhunderts.-Aufklärung in den Baltischen Provinzen Russlands. Ideologie und soziale Wirklichkeit, hrsg. von Otto-Heinrich Elias in Verbindung mit Indrek Jürjo, Sirje Kivimäe und Gert von Pistohlkors. (Quellen und Studien zur Baltischen Geschichte, Bd. 15.) Köln; Weimar; Wien 1996, S. 229-253; Raimo Pullat, Vermögensnachlaß des Seeligen Stadt Chirurgi Andreas Arnold Ritter, anno 1767. In: Acta Medico-Historica Rigensia, Vol. VI (XXV), Riga 2002, S. 11-21; Raimo Pullat, Nachlassverzeichnisse zweier Tallinner Handwerker im 18. Jahrhundert. Schneidermeister Johann Andreas Cellarius (1750), Schustermeister Christian Friedrich Blanckenburg (1770). In: 
Vana Tallinn XV (XIX), S. 179-196; Siehe auch: Raimo Pullat, Die Stadtbevölkerung Estlands im 18. Jahrhundert.-Veröffentlichungen des Instituts für Europäische Geschichte Mainz Abteilung Universalgeschichte, hrsg. von Heinz Duchhardt, Beiheft 38, Mainz 1997.

3. Die Nachlassverzeichnisse der Einwohner der Stadt Pernau, 1702-1800. Bearb. von Raimo Pullat, Tallinn: Estopol 2005 463S; Siehe auch: Raimo Pullat, Einige Entwiklungszüge der Wirtschaft und der Einwohnerschaft von Pernau im 18. Jahrhundert. In: Deutschland und Europa in der Neuzeit. Festschrift für Karl Ottmar Freiherr von Aretin zum 65. Geburtstag. 1. Halbbnd. Hrsg. von Ralph Melville, Claus Scharf u. a. Veröffentlichungen des Instituts für Europäische Geschichte Mainz. Abteilung Universalgeschichte. B. 134, 1. Halbbnd, Stuttgart 1988, S. 299-313.

4. Die Pernauer Ratslinie, von Heinrich Laakmann, Pernau 1926, S. 43.

\section{Inventarium über des Herrn Rahtsv. Lütkejohanns Nachlaß in praesence des Herrn Rahtsverwandten Heno geleget d 14 Juny. Anno 1714*}

\section{An Gold}

21 Krohn Golden Ring mit

3 kleinen echten Steinen.

1 dito mit 3 blauen Steinen

ein gedreheter Golden Ring

1 Bretschen

obige drey Ringe und Bretschen sind bey Herr Hans

Bucholz versetzet:

goldene Ringe bey Magnus

Hinrich Grabbe versetzet.

\section{Silber}

Drey silbern Löffel, eine Balsam Büchse und des seel. Rahtsverw. Bildniß u. silber vergult. stehete bey $\mathrm{H}$. Hans Bucholz versetzt.

4 Leffel. 1 Paar silberne Schnallen, ein Stock Knopf, und eine Balsam Dose wiegen zusammen 23 loth $1 \mathrm{qt}$.

5 neue silberne Leffel der Frau Rahtsverwandtin zum Hochzeit Geschenck verehret. Wiegen zusammen 19 loht.

Ein Paar Ärmel Knopfe wiegen
3 qt.

Ein silberner getriebener Krug.

Deckel an denen Ränden vergüldet 8 1/2 loht.

2 Reihe Agtstein Corallen.

1 schlechte kleine Taschenūhre.

\section{Zinnen}

9 gebrauchte Zinnerne Schüsseln mit 3 Zeichen wiegen $2 \mathrm{Llb}$

11 gebrauchte dito Teller wiegen $15 \mathrm{lb}$

2 Paar Zinnerne Leuchter wiegen $61 / 2 \mathrm{lb}$

Alt Zinn $2 \mathrm{Llb} 4 \mathrm{lb}$

Ein Zinnern Handfaß wieget 2 Llb $71 / 2 \mathrm{lb}$

\section{Kupfer}

Zwey destillir Kessel mit eisernen Füssen und Ohren, dabey ein Pfeiffe $1 \mathrm{Llb} 10 \mathrm{lb}$

3 Kessel, eine Pfanne mit eisern Griff und eine Caffe Kanne $1 \mathrm{Llb} 10 \mathrm{lb}$

Ein alter Helm wiegt 2 1/2 lb
Alt Kupfer $3 \mathrm{lb}$

Metall

1 metallen Grapen mit 3 Füssen wieget $12 \mathrm{lb}$

Ein Streich Eisen.

Ein metallen Leuchter wieget $11 / 2 \mathrm{lb}$

Alt metall $12 \mathrm{lb}$

Messing und Blech

Ein Messing Grapen mit 3 Eisen

Füssen und eisernen Griff $71 / 2 \mathrm{lb}$

Ein Durchschlag mit eisern Ohren

3 Apothecker Kessel mit eisern Griffen

wiegen $81 / 2 \mathrm{lb}$

2 Messing Grapen mit eisernen Griffen wiegen $5 \mathrm{lb}$

Eine Messing Feuer Pfanne und

Kessel mit eisern Ohren wiegen $41 / 2 \mathrm{lb}$

Ein Messing Becken wieget $6 \mathrm{lb}$ Ein Paar getriebene Messings 
Leuchter wiegen $21 / 2 \mathrm{lb}$

1 Messing Caffee Kanne und

1 dito Thee Kanne wiegten

$5 \mathrm{lb}$

Ein Messings Licht Schere.

Ein blechern Armen Büchse

Ein blechern Lachs Pfanne

1 mittelmässiger Trichter

4 blecherne Thee Kannen.

3 zerbrochene.

\section{Eisen Zeug}

2 Braht-Spieße

1 Knecht dazu

Eine längliche Braht Pfanne

1 runde dito

Eine Röste.

2 Feuer Zangen.

1 Feuer Gabel.

3 eiserne Licht Scheren

Eine eiserne Elle.

3 Waffel Eisen.

1 groß eisern Dreyfuß

3 kleinere

Eine eiserne Ofen Tühre

Ein eiserner Grapen

Einer Papier Schere

\section{Leinen Zeug}

17 Manns Hembde

41 flächsen Bett Lacken.

55 mit Küssen Bieren theils mit

Spitzen, theils mit Bunten.

15 drellen Handtücher.

20 kleine dito von schlechter

Leinwand.

39 drellen flächsen Tafflacken.

10 grobe dito

54 drellen Servieten

4 getragene Manns Halßtücher von Nesseltuch.

1 von Slesischer Leinwand.

4 Paar Manschetten.

2 halbe Hembde von Wahren

Dorffer Leinwand.

8 ordinaire abgetragene

Schnupf Tücher.

2 Spitzen Nacht Kappen.

2 durchgezogene dito.

7 Blatter zu Kissen Bühren von Spitzen

1 ausgenete dito.
6 1/2 lb Knucken Flachs

Bett Zeug

9 Unter Pfühle

4 Haupt Pfühle

21 Ohrkissen.

Ein Cantunen Bettdeke ungefuttert.

Eine blaue scharsen Gardine.

Eine blaue raschen dito.

Eine gedruckte Heden Drellen Gardine.

Ein bunter Bett Helffer.

\section{Kleider}

Ein neuer Manns Rock vom schwartzen Lacken mit gestrifften Atlass gefuttert, dazu Cammisohl und Hosen von Dammasch.

1 neue Unter Cammisol von gelben Attlass.

1 dito von Meer grühn Dammasch.

Ein alter brauner Rock mit Fuchs gefuttert

\section{daran die Knopflöcher mit} von Silber Tressen besetzet.

2 alte schwartze Röcke, und 2 dito Cammisöle

1 Paar alte Hosen von schwartzem Triep.

Ein grau Cammisol von Scharße.

3 alte Schlaff Cammisöler von Cantunen.

2 alte dito Schlaff Röcke.

Ein blauer Lackens Mantel.

2 Mund Kissen von Cantunen

Eine getragene Alter Mütze.

Ein getragener Hut.

2 Paar alte schwartze Strümpfe.

2 Paar alte grühne.

Eine weiße getragene Perique mit Alongen

Eine braunlichte kurtze dito

Eine alte weise dito.

1 Paar Schuhe von blanck Corduan.

1 Paar von rauch Corduan.

1 grühne und weiße Manns
Scherffe.

Eine weiß und blaue dito.

Eine weiße von Zwirne.

Ein leicht brauner Frauen Peltz mit Marder Kehlen, und Fuchs gefuttert mit Golden Tressen von der Brust und umb den Armeln besetzet.

Eingetragenes blau bordirtes Seiden Futter Hembd.

Ein gestriffet Attlasch Frauen Cammisol.

Eine schwartze dammasch Frauen Scherff.

Eine dito von Bordelu.

Eine rohte Sammeten Mütze mit Zobeln.

Eine schwartze Sammeten Kappe mit Bordelu.

Ein schwartzer Unterrock von Dammasch mit Einer Silbern Tresse 3 Finger breit

1 schwartzes Mund Kissen von Dammasch.

Ein gestreift Seiden Rock ohne Querll.

3 Zobeln Muffe.

1 Paar alte schwartze Seiden Handschu mit Golde bordirt

Ein alter Muff von Luchs mit einem silbern Ring

Ein schwartzes kurtzes Vortuch

1 rohtes dito.

1 gelbes dito.

Eine schwartze Sammeten Masque.

Eine silberne Leib Borte 1 1/2 Finger breit und Seiden Band gefuttert und einen Zopf von Golde Frentzen.

Eine Brust Latze mit Gold und Silber durchwirket.

1 schwartze dito mit Silber ausgeneet.

Eine Seiden Tischdecke.

2 Paar weise lederne Handschuh.

1 Paar rauhe Frauens Handschuh.

1 Paar rohte seidene Frauen 
Strümpfe.

11 Ellen schwartze Spitzen.

$51 / 2$ Ellen grühn und roht Band 3 Finger breit

4 3/4 Ellen schwartz Band 3 Finger breit

4 Ellen grühn Band mit Silber durchwürket.

6 Ellen geblühmt weiß und roht Band 4 Finger breit.

Ein Rüstung weiß Flohr

Eine schwartze Flohr Kappe.

5 Ellen Haartuch.

Eine seiden Nadel Kisse

Ein weiser Frauen Unterrock von Drell

Ein weiser Spitzen dito

Ein grühn Kutschboyen Unterrock.

Ein alt schwartze Flohren Mütze

Ein Stufe grob braun Lacken.

1 Stück von bereiteten Elend Leder.

Ein Paar weiße Manns Handschuh.

Ein Paar alte brauhne dito.

Eine groses Nadel Kissen.

Eine Cantilie Spitz untern Rock.

Ein Paar doppelte Frauen Manschetten mit Spitzen

1 schwartze Bordeluen Kappe.

Gewehr, Montirung, Seilen

Fahrzeug

1 Paar Dragouner Pistolen.

Ein Messinger Gefäß, ein halben Stichblad und silbernen Griff.

Ein dito mit eisernen Gefäß, welches mit Silber eingelegt.

Eine alte Musquete

Ein alter Sattel mit hinter und vorder Zeug.

Ein Paar alte Spohren.

Eine Vorseile mit Messing beschlagen.

Eine rohte Schlitten Kissen von Triep

Ein verguldeter Jagd Schlitten. Eine alte Vorseilen.
19 Schellen zum Jacht Schlitten.

3 alte Baur Schlitten.

1 Borck Schlitten.

1 Baur Wagen

1 unbrauchbahrer dito.

Moeublen, Haußgeräht, und ander Zeug

Eine turkesche Tapete.

Eine eichene Bett Stelle

Ein brauhn vermahlter runder Tisch.

11 mit rohtem Jucht beschlagene Stühle.

1 Kleiner dito.

Ein groser bunter Lehn Stuhl.

Ein Schreib Pulpet von Eichen Holtz.

2 verguldete langl. Schachteln in den 1 alte Corallen liegen.

2 runde dito.

1 vierekter weiser Tisch.

Ein Nacht Tisch.

Ein brauner Futterahl zum Hand Faß.

Darunter ein Schapf.

Ein brauhner Kleider Schranken mit gelben Leisten und 2 Tühren

Noch einer mit 2 Tühren und angestrichen

1 weiser Speiß Schapf.

1 Wand Uhre.

Ein mittelmäsiger Spiegel mit ein schwartzen Rahmen

Ein kleinerer dito

Noch 1 kleiner dito.

Das Holtz, darin die Handtücher Hengen.

Ein groser Dantziger Kuffer.

Ein anderer dito.

Eine weiße langlichte Lade

Ein grühner Dantziger Kuffer

Ein Flaschen Futter mit 4 Flaschen.

Ein Schirm mit 6 Blättern vermahlet.

Ein Schlaf Banck.

Eine Kinder Wiege.

2 Flasch Futter ohne Flaschen.

1 dantziger Schreib Lade, so unfertig

Ein groser Kasten.

2 kleine dito.

Ein Periquen Stock

1 Paar Messer und Gabel mit

Helffenbein Stilen

1 kleiner vierekter Lade.

1 schwartzer Kasten.

1 vierekter Kasten.

1 groser Baur Kasten.

3 Paar Woll Kratzen die noch nicht auf Bretter geschlagen.

Ein langl. Mitzen Schachtel

$91 / 2$ st getrucknet Ochsen-

leder.

11 Verdorbene Kalofeln

1 par holtzer ranken.

\section{Pferde und Vieh und Feder}

Fasel

1 braunes Pferd mit ein stumpfen Schwantz.

1 schwartze Kuh

1 Calicutscher Hahn.

1 anderer Han.

3 Hüner.

NB! Das braunes Pferd ward

d 23 Juny besehen und befunden, daß es gantz und gar aus dem Futter, weil sich uns niemand zur Auffutterung des Pferdes verstehen wolte, beliebte uns es auf 12 Carol. hochstens zu taxiren und demjenigen der es dafür anstinde zu überlaßen, der Diener Forstman erbot sich, die 12 Carol. zu geben, also wurde das Pferd wegzunehmen

\section{Stein und Gläser Zeug}

Ein bunter Krug Bluhmen darin gehalten werden.

Ein bunter Bier Krug mit einen Zinnen Deckel.

Ein brauner Bier Krug

3 Bier Gläser

6 Spitz Glaser

1 Thee Fläschen.

8 kleiner Brantweins Gläser 
6 Thee Köpfe und Schalen

5 Schalen

Eine barbier Schale.

1 steinerne Hollandische Schüssel

2 Senff Schalen

12 steinerne Teller.

1 Kalte Schal Kauß.

1 Große tiefe Hollandische Schüssel

6 Kraut Töpfe, und soweil Schalen darunter

Apothecker Wahren und Instrumenta

1 1/4 lb Radicis Angelicae Silvestris.

1/4 lb Radicis Alkannae.

3/4 lb Radicis Altheae.

$13 / 4$ lb Radicis Aistologiae longae.

$13 / 4 \mathrm{lb}$ - rotundae.

2 1/2 lb Radicis Aronis.

4 loht Radicis Asari.

4 loht-Aspargi.

1/2 lb-Bardanae.

$3 / 4 \mathrm{lb} 3$ loht radix Been. rub.

1/4 lb-Brusci.

3/4 lb-Calami

1/4 lb Carlinae

12 loht-Chinae.

12 loht-Cichorii.

$1 \mathrm{lb} 12$ loht-Consolidae.

\section{lb loth}

2 Cizeri rotundi.

126 Diptami rotund.

14-longae.

3/4 Doronicae.

312 Enulae.

1 felniculi.

20 foenicus Venetianae.

4 1/2 Galappae.

22 Grios. florent.

$1 / 2$ Radicis Imperatoris

4-liquiritiae

5-Pentaphilli

12-Peucedani

6-impinellae

3/4-Polypodii.

20-Rsaponticae

2-Succisae.
2-Squillae.

10-Tormentillae

$10-$ Victori alis rotundae.

6 -Victori alis verae

1/2-Ulmariae

3/4-Zedoariae

23 l.-Galangae minoris

12-Galangae majoris

13-Rhabarbari veri

3 3/4 erbae Alchymill.

5-Abrotarii.

3/4-Betonicae.

1-Veronicae.

1 1/4-Pulmonariae.

1/2-Basilicae.

6-Trifolii.

1 1/2-Melilot.

1/2-Hyssop.

1-Salviae.

1 1/2-origanii

3/4-Pulegiae

1-Centauri minoris

1 1/4-foliarum Senna. Hat H Rahtsv. Heno zu sich genommen.

$11 / 2$-Sabinae.

12-Nepethae.

8-Matrisilvae

5 Herba Gallejae

12-Marrubii

2-Paradisi

5-oliorum Lauri.

10-absmth. pontic.

4-Capillorum Veneris

1-Thimi.

6-Prunellae.

1/4-fiderae.

12 Herba Ceterach.

1 1/2 Adiantum aureum

13 Florum Balanstiae

5-Borraginis

4-Anthos.

9-Calendulae.

10-Buglossae.

3-Carthami.

8-meliloti citrini

12-meliloti albi

2-Lavendulae

14-Schüssel Bluhmen primula veris gantz alt.

13-Peoniae

1/2-rosarum.
1/2-Sambuci

5-verbasci

9-Csamamillae Romanae

14-Stechados Arabicae.

6-mari veri.

6-Spicae Celitae.

1-Diptami

3/4-Pelegii Cretic.

\section{Gummata}

lb Loht

14 -Aloes hepaticae

27-Gummi Ammoniacum

1 15-Gummi Animae

6-Assa foetida

5 13-Gummi Arabicum

5-Gummi Benzoes

NB. Gummi Asphalt.

3-Bidellium

1 6-Camphorae. Hat

H. Rahtsv. Heno zu sich genommen.

4-Gummi Elami

3 1/2 Gummi Galbanum

3 1/2-Laecae.

1 1/4-Ladan.

5-Myrrhae.

2-Mastygis

4-loht Gum. Sayepen.

1lb 12 1.-Copal.

1-3 1.-Sarvocolla

14-Caranna

3-Opoponaces

3 loht-Thragagant halb

9-Opii.

2-Sandaracae

19-Sangvinis Draconis

10-Sangvinis hirci

8 Scammonium

26 Succinum citinum

12 Storax calamita

18 Tacamahaca.

\section{Ligna}

lb loth

7 Lignum Aloes.

6 Lignum Buxi.

18-Colubrinum.

5-Cupressae.

9-Sanctum. H. Heno zu sich genommen.

17-Lentisci. 


19-Santali albi.
2-rubri.
11-Rhodii
13-Sassatiae. Hr. Heno zu sich
genommen.
14-Nephriticum

\section{Cortices}

10 Cortices aurantiorum

7-Citri

9-Thamariser

7-Chinae de China

116 -viscus queromi

11-ranatorum

\section{Semma}

lb Loth

239 Semen Anethi.

26-Ammeos.

1 16-Apii.

14-Aquilegii

3-Amomi.

3-Cardui Mariae

2 1/2-Cardui benedict.

25-Agni casti

1-Cynae.

8-Cydoniorum.

23-Cocognydii.

3-Nasturtii

7-Citrulli.

6-Cucumeris

15-Levistici

10-Dauci silvestr.

2-Dauci Cretici

13 -foeniculi Cretici

19-Petroselini Macedonici

1-Semmis Siberis montani

6-Papaveris albi.

11-Milii solis.

1 10-Nigellae. H. Heno zu sich genommen.

14-Staphis agrii

28-Paeoniae.

16-Tlaspios

4-Saturesae

3/4-foeniculi

15-Cummi

14 Unicornu Fossile

118 Lapis Albaste.

3/4-Magnetis.

4-Carniol.

10-Nephritici
2-Lazuli.

7-Ostiocolli.

7. Unicornu

4 Lapidis Spongiae

12 Lapidis Alabasti praeparati.

7 Lapidis Calaminaris.

9 Lapidis Granati.

8-haematidis.

11-Hyacinti.

8-Lazuli.

1 1/2-Rubini.

$31 / 2-$ Saphir

3-Smaragd

1 30-Percarum

1-Succini praeparati

312 Cornu Cervi Philosophice praeparatum

1/4 Cornu Cervi ustum.

5 Boli Aemoni praeparati.

26 Corallorum Albirii praeparat.

22-rubror.

8 Cranii humani Cipporat.

2 Lapidis camori Gipti

2 mandibolorum Lucii pptor

3/4 Lapidis Prunellae

3 Spodii

3 Tutiae.

5 Lapidis aquilae.

1 Lapidis Bufonis

20 Antihectici Boteri alt.

6 Antimonii Diaphoretici

7. Bezoardici animalis.

6 vitri antimonii

8 Crovi Martis aperient.

13-astingentis.

1 1/2 florum Antimonii.

17 florum Sulphuris.

3 1/2 florum viride Aris

9 Crovi metallorum.

3 Magisterium Diaphoret.

4 Magnetis Arsenicalis.

10 Mercurii dulcis

11 Hepar Antimonii

$11 / 2$ Mercurii pracipitati albi.

8 Nitri Antimonii

8 Sachari Saturni

1 1/2 resinae Guasaci

1 1/2 resinae Galappae. $\mathrm{Hr}$

Heno zu sich genommen.

1 loht resin. agariae

20 reguli Antimonii
2 1/2 Tartari Emetici

7 Vitrioli Martis

20 aris usti

6 Seminis Licopodii

6 Trochisci de Squilla

2 1/2 Aluminis plumosi. $\mathrm{Hr}$

Heno zu sich genommen.

20 Rasura Succini

6 umbilici marini

26 Lac Lunae.

8. Species pro Cucupha.

1 Species pro moesulis Imperatorum

17 Trochisci Bechici nigri.

4 Trochisi Alhandali

6 Pulveris aciidentalis cum apio.

11/2-Sine apio

4 Pulvis Carminativus

6 Pulvis contra Casum

2 Pulvis ad confortardum memoram

$11 / 2$ Pulvis dentifricius

7 Pulvis dyssentericus.

$11 / 2$ Pulvis epilepticus.

2 1/2 Pulvis Gutteta

1/2 Pulvis Pannonicus

9 Pulvis puerorum citrinus

8 Pulvis stomachalis resolvens

1 1/2 Pulvis Trivera

5 Diagridium Sulphuratum

5 Cremor Tartari

1 1/2 Species Dianthos

2 Species hyera Pierae Sine

Aloe et Agariae

3 Species Diagalangae.

1 1/2 Species Diamercurii

1 qtl. Trochisci Moschardini

1 loht Pulvis ungulae.

1 loht Lumbricorum terrestrium

4 fructus Thamarintorum

2 1/2 Spermaticis Ceti

1 1/4 Lapis befrar. occidentaly

31 . Iquentus Ol. Nucostae.

Ii. Lacc. florentia.

1 Muschel mit Silber

$1 \mathrm{~d}^{1 / 4} \mathrm{k}$ kleiner ohne Silber

1 Büchse mir Ziebeth. wiegt bruto 1 loth.

14 loth Maces.

4 Cardamomi majoris 
9 Cariophyllorum.

5 Anthophyllorum.

$1 \mathrm{lb}$ Piper longum. bruto

30 Manna. Hr Heno zu sich genommen.

2 lb Boli Armeni.

11 Granii hominis crudi

1 1/2 Terra Tripolis

31 . Nefetta nilva.

$1 / 2 \mathrm{lb}$ Borax Veneta.

2 lb Vitrioli de Cypro

$1 \mathrm{lb} 6$ Succi Acatiae.

22 loht Mumia.

14 loht orleans.

1 loht Myrobalini v. Emblio.

26 loht Lapidis Percarum.

6 fell vitri.

$1 \mathrm{lb} 30 \mathrm{~L}$. margahetae.

$1 \mathrm{lb} 12 \mathrm{~L}$. vitrioli albi.

20 loht Costis arabicus

24 loht Terra ferr.

6-Grana Paradisi

2 1/4 lb Sulphur vivum.

$13 / 4$ lb Schirbur weiß.

18 loht Torna solis

9 loht grana Chermes

1/2 lb Zuick

6 Cinnabaris

18 Glacies Mariae.

1/4 lb Storax liquida

$1 / 2 \mathrm{lb}$-gantz alt.

12 loth pulvis fumalis.

$1 \mathrm{lb}$ Succus liquiritiae. $\mathrm{Hr}$

Heno ebenfals zu sich genommen

$1 \mathrm{lb} 6$ loth nihil album.

$63 / 4 \mathrm{lb}$ Cerussee.

22 loht Terra Catechu.

25 Stk. Ungulae Alcis.

$9 \mathrm{lb}$ Sulphur Grysium

$4 \mathrm{lb}$ Victriolum communae

5 1/2 lb Zinziber alb.

3 1/2 lb lapis Pumicis.

$19 \mathrm{lb}$ bacc: lauri.

23 lb Alum: crud:

$13 \mathrm{lb}$ tarl. rubr.

$5 \mathrm{lb}$ brasil. coerul:

$12 \mathrm{lb}$ buccae Juniper.

$42 \mathrm{lb}$ Sulph. Citrinae

$12 \mathrm{lb}$ Semen anisi welche

H. Rahtsv. Heno zu sich

nahm mit condition zu zah- len oder in natura wieder

zu liefern.

3 1/2 lb Semen foeniculi. dito auf vorige condition zu sich genommen.

$3 \mathrm{lb}$ Cubeb.

$1 \mathrm{lb}$ oculi cuneri.

6 Stk. denter aperi.

$1 \mathrm{lb}$ Mandibul. luni piscis.

1 klein stück Helfenbein.

$71 / 4 \mathrm{lb}$ diverse sorten terce sigillatae

5 3/4 lb Grichische Seiffe.

20 loth alum. Sacharinum

5 loth Semen plantaginis

5 loth Sem. coruli

$1 \mathrm{lb} 10$ loth Sal amoniacum

$13 \mathrm{lb}$ herbae anthol. H. Heno zu sich genommen.

21 loth Semen raparum.

10 loth grana paradyli

8 loth Lap: osteocoli

8 loth Piper: Alb:

8 loth terra victrili dulcis.

7 loth Spernioli crolis.

4 loth coeruleum montan.

2 loth Viride montanum.

24 loth pulv: ocul. carcrorum.

16 loth pulv. Irios.

6 loth pulv. paeoniae

3 loht pulv. glyciricae

5 loth pulv. Myrrhae.

4 loth pulv. benzoes.

6 loth pulv cubebar.

3 loth pulv. ulmariae.

5 loth pulv. Zedicariae.

6 loth pulv. Zinab.

12 loth net extract. ment. cum vitro gewogen

2 loth pulv. Hepat. ruber

14 loth extract de aqua apoplect cum vitro gew.

11 loth extract theriacal cum vitro gew.

$2 \mathrm{lb}$ nitrum antimon.

$2 \mathrm{lb}$ schwartz lack.

11 loth Cera alba.

$1 \mathrm{lb} 14$ loth rubrica fabrilis

$1 \mathrm{lb} 28$ loth Lupur hematitilis

2 1/2 lb Umbra

$2 \mathrm{lb} 4$ loth Cortices timiamates

16 loth roth Holtz.
20 loth Fernebock.

$2 \mathrm{lb} 4$ loth pulv. eqvotum.

$15 \mathrm{lb}$ bolus alb: et niben

$10 \mathrm{lb}$ ockergelb.

12 loth Xiliqva dulcis. nuhr nutz

$1 \mathrm{lb} 8$ loth fructy Sevestes gantz verdorben

16 loht Colla pehium

28 loth gallo turcae

14 loth iundelae fumales.

5 loth aquariig crudus.

1 loth Ol. Carvi.

2 loth Ol. Anthos

1/2 Drach. 3 1. ol. Anisi

1/2 Drach 3 1.-Chamamom u. vulg.

5 loth Ol. Caphera crall.

2 Drach 3 y Ol. Caryephyllorum.

3 loth Ol. Cumini.

1 1/2 Drach. 3 iß Ol. Aneth.

2 loth Ol. Sabinae.

2 loth Ol. Menthae.

1 loth-Calami aromata.

3 Drach-Lavendul. Hr. Heno zu sich genommen.

12 loth Ol. Cerck.

1 loth-Juniperi ex beceei. Hr. Heno zu sich genommen.

1 Sempel Ol. Cinamomi

3j Ol. legi Thod.

4 loth Balsam. Indic. alb.

6 loth Stall. Sulphuris terebinthinata

6 Drachma. Ol. Succin. Citrin.

3 3/4 lb Ol: Juniperi ex ligno.

$\mathrm{H}$. Heno zu sich genommen.

$3 / 4 \mathrm{lb} \mathrm{Ol}$. petrae.

8 loth Ol. Castory

3 Ol. Mastiqel

2 loth Ol. papaveris

2 loth Ol. tarteri per deliqvuus

8 loth Ol Scorpion:

4 loth ol. hyoziami

2 loth ol: tart. faetid.

1 1/2 loth bulfom. Arceae.

3 loth ol. phifosophorum.

$1 / 2$ lb ol. Sperm Ranariin

$1 / 4 \mathrm{lb}$ ol. Caporum.

1/2 lb ol. Terebint. H. Heno zu 
sich genommen.

2 Kruge worin olea cocta anonyma.

$1 / 2 \mathrm{lb}$ axung. hominis.

$1 / 4 \mathrm{lb}$ dit. gallino.

$1 / 2$ quent. Schlogbalsam.

1 1/4 Kerl Hr. Heno zu sich genommen.

$1 \mathrm{lb}$ Spirit anthos.

$1 \mathrm{lb}$ Spirit cochlear.

20 loth Spirit angelic.

4 loth Spirit. ceraforum

$1 \mathrm{lb}$ Spirit vini camphoraty

3/4 lb Spirit cnastygis.

18 loth Spirit. lumbricorum

5 loth Spirit formicorum

$1 \mathrm{lb}$ Spirit cranii humani.

1/2 loth Spirit sangvinis humani.

1 1/2 lb Spirit Rosar.

4 loth Spirit Nahturii

2 loth Spirit thercacolis

3/4 lb Spirit Juniperi.

1/4 lb Spirit Salis amoniaci

1/4 lb Spirit Tartari

4 loth Spirit. Sulph. Terebentinaty.

2 loth Sp Salis crudi

4 loth Sp. Salis dulcis.

16 loth Spirit Sulphuris

8 loth Spirit rerinae

$11 / 2$ sal volatile oleosumSylvii

$11 / 4 \mathrm{lb}$ clyssi antimony.

$1 \mathrm{lb}$ aqva viridil

$1 / 4 \mathrm{lb}$ aqva mercuriulii

1 loth Spirit balsamicus.

4 loth blau augenrogter.

$1 / 2$ stoff anhaltina

4 stoff aqva apoplectica.

3 loth aqva fortis

$11 / 4 \mathrm{lb}$ aqva regiae

$3 / 4 \mathrm{lb}$ phlegma a Sale volat oleoso

$1 / 2 \mathrm{lb}$ aqva calcis vivae.

20 loth tinctura bezoard. Michelis

8 loth essent. galangae

$1 \mathrm{lb}$ tinctura laccae.

$1 \mathrm{lb}$ elixir ante febrile

1 1/2 loth laudanum liqvidum

5 loth elixir pectorale Regis
Duniel

2 loth elixir proprietatis

8 loth elixir vitrioli Mynsiehtii

$11 / 2$ loth essent. cassiolignea

$1 \mathrm{lb}$ Ess. Calami aromatici.

8 loth essent. auranciorium

4 loth tinctura Croci.

2 loth essent. carnimativa

16 loth essent legni Sancti

$11 / 2$ loth essent. Castorii

12 loth essent. Gentiaro

6 loth essent. Myrrho

4 loth essent. hypocratis

4 loth essent. Menthos

$1 \mathrm{lb}$ essent legnorum

8 loth essent. pini

3 loth Infusio essentio lumbricorum

6 loth tinctura odontalica

2 loth butyrum antimony

21 loth diverse couleur flunkert.

$1 \mathrm{lb}$ Mercurius vivus

3 qvint. Saffran

Eine kleine Lundislade welche halb vol mit ungelöscht kalck ist

$1 / 4 \mathrm{lb}$ conserva fumario alt.

$11 / 4 \mathrm{lb}$ conserva rosarum rubraru alt.

12 loth Chylonium romanum.

16 loth Vieh theriae

8 loth passulo luxativae alt.

$1 \mathrm{lb}$ vih de pulnione vulpis.

16 loth Syrup cardo benedicti

Eplastra

$11 \mathrm{lb} 20$ loth Kleplechtru alb. coctum

28 loth de baccis lauri

$1 \mathrm{lb}$ de bethonica

6 loth drachyl. c. Gummatibus

16 loth drachyl Simplex

25 loth Citrinum

22 loth de crusia panis

18 loth Grysium

7 loth ad hernias

$1 \mathrm{lb} 28$ loth de mynio

18 loth de meliloto

$1 \mathrm{lb} 6$ loth de galbano

$1 \mathrm{lb} 12$ loth de fensivum

8 loth Uppadeldoch
8 loth Ovierocium

5 loth de Spermate Ceti

24 loth de asulphuris Rulandi

2 loth stomachale

10 loth vesicatorium

$2 \mathrm{lb}$ Cera arborea

14 loth roth sergel Wachs

8 loth Gely Wachs

22 loth Confect. Sem. foenicul.

5 loth Cem. Cynae

$1 \mathrm{lb} 12$ loth Sem. Coriandr.

$26 \mathrm{lb}$ passulo Maj.

6 loth pulvis tabais

1/4 lb unqvent Aegypt. u. zwey

Krüge.

$1 \mathrm{lb}$ unqvent alb. camphorat.

$11 / 2 \mathrm{lb}$ dit Simplex

$1 / 4 \mathrm{lb}$ de althea in 2 Krügen

$1 \mathrm{lb}$ unqvent. pomatrium alt.

4 loth unqvent. apostolorum.

$11 / 4 \mathrm{lb}$ unqvent. pedunlorum

2 Krügen

$1 \mathrm{lb}$ unqvent digestivum

$1 / 2 \mathrm{lb}$ unqvent alabastrinum.

$21 / 2$ unqvent contr. Scabium

$1 \mathrm{lb}$ unqvent portab. rubrum

6 loth unqvent. Mercuriale

20 loth unqvent Nutritum

$1 / 2 \mathrm{lb}$ unqvent ad haemorroides

$1 \mathrm{lb}$ unqvent. calendulae

$1 / 2 \mathrm{lb}$ unqvent. Nihili ad oculos

Ein Krug worin $1 \mathrm{lb}$ unqventum Anonym.

$5 \mathrm{lb}$ Oleum laurini.

1/2 lb Roob Sarmduis.

Ein tonchen mit alt Schmaltz.

Ein Glaß cum infusione floru liliorium coevalium.

$2 \mathrm{lb}$ Spirit corn u Cervi. crudi.

$1 / 2 \mathrm{lb}$ hal. corn. Cervi. crudi.

4 loth unqvent Stomachale

4 lb Spirit vinia rehina Galappi avocati

$1 \mathrm{lb} 12$ loth merc. Sublimat.

$23 / 4 \mathrm{lb}$ arsenicum album

$11 \mathrm{lb}$ arsenicum rubrum

3 3/4 lb Citrinum

$83 / 4 \mathrm{lb}$ aurum pigmentum.

$1 / 2$ loth anibr. liqvid

Ein Kasten mit alte pillen 
Massen.

$1 / 4 \mathrm{lb}$ eingemacht Ingfer.

$1 \mathrm{lb}$ Carsia lignea.

30 loth pulv. pro. Elect. Orvietan.

$1 \mathrm{lb}$ pulverisirt Toback.

\section{Aqua Compositae}

$11 / 2$ stoff Aq Cordialis

1 1/2 stoff Aq Samascena

2 stoff Aq. vit. mulierum.

Infusiones V Epilepticae

- V Cephalicae

\section{Simplice}

2 1/2 stoff Aq. Ivsar.

1 stoff-Melissae alt

1 stoff-Salinae alt

$11 / 2$ stoff-Calaminthae alt

4 stoff-Plantagirvi gantz Dick

3 stoff-Menthae alt.

6 stoff V Hyssopi alt

2 st. alt Syrop Violar

2 st. Aq Storragini alt

$11 / 2$ st.-Anthos gantz alt

1 st. Aq. frenioni alt

1 st.-Selivae alt

1 st-Juniperi

Ein Halb Tonne mit eis. Nadeln Netto gewogen 5 llb 15 $\mathrm{lb}$ so der $\mathrm{H}$. Heno zu sich genommen.

$1 \mathrm{Glaß}$ mit ein nubelantes Waßer

56 st. Künwachs Büchsen

1 große Kruke so weniger dan Halb voll mit Theriac ohngeschlagen

$8 \mathrm{lb}$-hat H Rahtsv. Heno zu sich genommen

1/2 Tonne die ohngestr. Halb voll:

14 Buch. schlecht Schreib Pappier

1 Buch. groß Post Papier

$1 / 2$ Buch. ordinaire Pappier

2 Buch. weis Maculatur

57 Bogen allerhand gefarbt Pappieren

2 Vier u. neunzig Bucher schlecht Maculatur. $\mathrm{Hr}$ Heno zu sich genommen.
8 Bucher Gold.

5 Bucher Silber.

1 Karpe mit alten Brieff Oblaten

1 große eiserne Haußwage mit Holtzen ohnbeschlagen Wachs Schalen dabey 1 Gewicht.

22 loth Bierfade

1 Zinnern und

110 st. diverse Apotheker Büchsen.

1 angestrichen Apoth. Tisch mit 55 stk. Schupladen u. 2 st. Morser Blöcke.

1 angestrichen Repositorium mit 32 st. große Schupladen.

Noch 1 do mit 18 stk. do

Noch ein großer angestrichener Repositorium

1 alter Schappf mit 7 st. Schuplade

7 st. große \& kleine Schuplade

1 groß u. 1 kleine eisern Mörsell u 2 st. Keile.

4 alte Paar Apoteker Siebe

4 do alte Basten Siebe

1 groß u 1 klein eisern Mörsell u 2 st. Kruke v. Eisen.

1 groß metallen Mörsell u. 1 Keule

1 klein Meßing do

3 st. Serpentin Mörser mit 3 Keule.

2 Schachtel mit Grif und klein Gewicht

1 Geld: Gewicht

7 st. alte wagschale

2 st. felle

Einige Sätze holtzerne Apoth. Schachtel.

2 Brieffe mit Baur flitter Geld.

4 Spatels

1 Tenaculum

1 höltzern Plaster Bret

4 st. zinnen Apoteker Maße.

1 Presse mit 2 eiserne Platen

1 apotek: prupor. Stein

5 Erne Retorten

5 Kolben, darunter 3 alte

5 gläßerne Retorten, worunter 3 alte
4 Hübner

2 alte kleine Pfiolen.

1 alt groß Recipient

12 Stroh Wein u. Brandtwein Röhmer von ein halb Stoff bis $1 / 2$ qtin.

30 Stroh Apoteker Gläßer

11 1/2 Stroh Ungrisch Waßer Gläßer.

1 anders Lade mit alte $u$. neue, groß und kleine Zeug, wie auch kleine Tirgels einige Salg.

1 groß alt Tirgel.

1-Juder Glaß.

8 stk. kleine kantige Juder Gläßer

100 stk. alte Gläßer.

1 alter Apoteker Kasten.

1 Tonn ohngeschl. 1/4 Anker voll mit gantz gemeine Baur Pfeiffen.

\section{Einige lange do}

An Büchern

In folio

Christoph. Achati Hageri Buchhaltery.

Lazari Riverii opera medica.

Osiandri Biblia Sacra.

Georg Daniel Koschwitz Arzneyschatz

Renberti Dodonnoi Herbarum.

Ein in turkisch Papier gebundenes Buch mit gedruckt Columnen.

Ehstnische Privilegien und andere Begebenheiten Msct.

Julii Belli Laurea Austriaca

Joh. Conrad. Gobelii Augustana Confessio.

Ein alt Lateinisch Lexicon ohne Titel.

Das halsche Kirchenbuch Msct. de Ao 1705.

Christ. Besoldi Thesaurus practicq.

Joh. Hermanni Postill.

Ein alt Buch darin einige Kupfer geklebet 


\section{In qvarto}

Ein volumen Disputationum.

Johannis Schlemnii Apostolische Rüstkammer.

Jacobi Ayreri Processus Belial contr. Jesum

Hercules und Valchea

Jo. Bart. Niemeyeri Symb: Apostol. wobey einige kleine Tractaten bey gebunden.

Copenhagener Apothequer Taxe.

Ernest Wilh. Prangen Pest Chur.

Joh Fridr. Mayers Evangelischer Engel

Wasmuthi Hebrachung

Nicol. Belli Kay serlicher Triumpfwagen.

Gerhardi Schola pietatis.

Christ. Phil. Richter de Sunephone $\mathrm{ab}$ intestato

Creidii Leichpredigten.

Ein Band von allerhand Tractaten

Jacob Raupii januae SS Theologiae.

Ein Band diversen Tractaten.

Predigten über der Buß Psalmen ohne Titul

Dedr. Reinkingks biblische Policey

Lieffländische Landsordre und Luendendorferi de actioniby Successoriis welches d. Post Zimmerman als das seine abfordern ließ

Joh. Zivel ven Dispensatorium

Schraderi Kirchen Formular

Drentii Casus Conscientiae ohne Tittul

Anonymi Curiosa Juris publ.

Ein Tomy von dem Actis naturae curiosorum.

Brandenburgische Apothequer Taxa.

Jac. Reinicii Clavis Theologiae

Happeliy von türk. Kriege.

Jh. Schmidts Buß Predigten

Conrad Mels Posaune der Ewigkeit.

Ein Band allerhand teutscher tractaten

Zach. Lundii deutsche Poematae

Bertii Comment. reru Germanicarum.

Ein alt Postill ohne Titul.

Sächsische Apothequer Taxa.

Kirchen Ordnung

Politische Schatzkammer ohne Titel

In Octavo

Andr. Hollanders Spiegel Guter u. Böser Regenten.

Anonymii Kunst u. Werkschule.

J. Meyers Frühstunden.

Reinii Logica.

Eine Lüneburgische Biebel

Joh. Bodiny de Republ.

Calvisii Lexicon.

Baglivi praxis Medica Teutsch

Calovii Theologia.

Czarischen Maytt. Lebens Beschreibung.

Philip Verheins Anatomie.

Cardeluccii Stad und Land Apotheke.

Werneri Homilia

Mayfarti Melleticcii Oratorien tom 2dy

Stanihurssii Historia de passione Christi.

Bertii Geographie

Chemnetii loca Theologica

Die Evangelien Grichisch Teutsch und Lat. ohne Titul.

Dexelii Theatrum Historicum.

Konigii Theologia et Donati Metaphysica

Corneliy Nepos.

Jacob Daniel Ernst Bilderhauß

Junkens Leibarzt.

Schmidii Hist. Ecclesiastica.

Calvisii Thesaury latini Sermoni

Anonymi curieuser patiente et Veslins Anatomia.

Nürenbergische Biebel mit silbernen Klammern

Moletorii Sermones parationes.

Welleri Grammatica Graeca.
Talanders Briefsteller.

Nov: Test. Graece et lat.

Schroderi Theologia

Christian Buchners Beschreibung von Pistri

Anonym. Außlegung über Evangelia

Conimoi Leben Ludewig des XI

Osiandi Controversien

Terentii Comoedien

Calvisii Lexicon.

Eine alte Rhetorica ohne Titel.

Mynsicht Hatz und Rüst

Kammer.

Thomasii Monaths Gespräche.

Erasmi Francisii Wohl der Ewigkeit.

Hosemannii Von Heil. Ehestande.

Graveri protectiones in August. Confessionen

Althammeri loca Scripti.

Meysneri Meditationes.

Habermans Niederteutsches Gebet Buch

Frantzii Hystoria Animalis

Schumans komische Schriften.

Digby Sympathia

Sluteri Logica Aristot.

Copie teutscher Helicon

Ein Platdeutsch Gebeth Buch.

Riedelini Medulla Charmacopoecae Augustano

Heilers Jesus Gedanken.

Seyboldi Grammatti

D. Joh. Fausti Lebens Beschreibung.

Fallopii Kunst Buch.

Guberti Ackerstudent

Ein klein Hollandisches tractatus ohne Titel

Ein Geschichts Calender ohne Titul.

Ein schwedisch Kreuter Buch.

Schoniß Reis und Hauß Apotheck

Ein alt Gebet Buch ohne Titul.

Ein alt medicinisch Tractat ohne Titul

Ein Gebeth Buch ohne Titul.

Fabulae Esopi. 
Ein alt colloqvium ohne Titul. Phil. Melanchton Examen.

Gartzii Lexicon Graeco Lat. Getzolii Lex. Graec. Lat. Waltheri Harmonia Script.

Rigischer Großer Cathechismus.

Florilegium Biblicum

Anonymii Carneval.

Hocus Pocus oder Fastenspieler

A. BC. von notis variorum.

4 st. Noten Bücher.

Ein lieder und leber Reimlein Buch Msct.

Lusart Pocken Meister.

Pfeifers von der lineber Natur

Juncken Lexicon Chymicopharmacenticum

In Duodecimo

Caroli XII Lebensbeschreibung

Beseli Schand des Politischen Glücks.

Ein teutsch Buch ohne Titul. Noch 1 dito
Arndts Paradieß Gärtlein.

Ein Gesang Buch alt.

Geistl. Handels Stab.

Ovidii Opera.

Leurdeni N. Test. Graec.

Justinius.

Schwedische Kriegs Articul

Kempe de imitat Christi

Sagitary Nucley Hist. Geom.

Ein alt schwedisch Buch ohne Titul

Ein lateinisch dito.

Neumans Kern aller Gebethe.

Flora Francica.

Aug. Pfeiffers Art Artium

Urb. Regii Dialogy.

Schefflers Himmelreich.

Beschreibung einer Ambassade an d. König zu Kain

Französische Hofflichkeit

Buchleri Thesaury Phrasum Poet.

Scharffi Logica

Tract. de Ebriatate

Rudolphi Sententia in dyplo

Neidenburgi lieflandischer
Landman

Ein alt klein Gebeth Buch

Das Evangelische Rostock

Keigelii 12 geistl. Andachten

Das Stockholmische deutsche Gesangbuch

Hypocrates in Sedecimo

5 Stück diverse Land Charten.

\section{An Schriften}

In dem Föhren Pulpet.

1 Schuplade mit allerhand Schriften darunten nichts sonderliches sich befand.

1 dit ebenfals nichts sonderl. darinnen

1 dit. worinnen ebenfals nichts als Brieffe.

Einige andere Lohn Schriften und Bücher.

6 st. theils Schuld theils andere Bücher darinnen der sel. Man eigenhandig geschrieben.

\section{Obligationes}

Von Carl Föebeter groß 23 Rtr 4 w. wobey eine Specification von dem was der seel Mann bey Grabbe versetzet.

Hinrich Haack revers von 5 rh a 80 Cop.

${ }^{\star}$ Estnisches Historisches Archiv, Bestand 1000, Verz. 1, Nr. 7342, Fol. 5-25.

Raimo Pullat, Dr. habil., Professor

Tina $21 \mathrm{~A}-1$

10126, Tallinn

Estonia

Tel., Fax +372 6485774 